\title{
OBSERVATION OF TeV GAMMA RAYS FROM THE UNIDENTIFIED SOURCE HESS J1841-055 WITH THE ARGO-YBJ EXPERIMENT
}

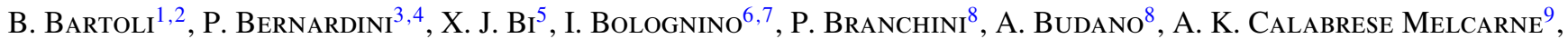

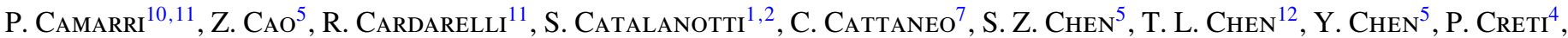
S. W. Cui ${ }^{13}$, B. Z. Dai ${ }^{14}$, G. D’Alí Staiti ${ }^{15,16}$, A. D’Amone ${ }^{3,4}$, Danzengluobu ${ }^{12}$, I. De Mitri ${ }^{3,4}$, B. D’Ettorke Piazzoli ${ }^{1,2}$, T. Di Girolamo ${ }^{1,2}$, X. H. Ding ${ }^{12}$, G. Di Sciascio ${ }^{11}$, C. F. Feng ${ }^{17}$, Zhaoyang Feng $^{5}$, Zhenyong Feng ${ }^{18}$, F. Galeazzi $^{8}$, E. Giroletti ${ }^{6,7}$, Q. B. Gou ${ }^{5}$, Y. Q. Guo ${ }^{5}$, H. H. HE ${ }^{5}$, Haibing Hu ${ }^{12}$, Hongbo Hu ${ }^{5}$, Q. Huang ${ }^{18}$, M. IACOVACCi ${ }^{1,2}$, R. Iuppa ${ }^{10,11}$,

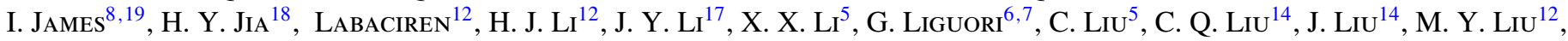

H. Lu ${ }^{5}$, L. L. MA ${ }^{5}$, X. H. MA ${ }^{5}$, G. Mancarella ${ }^{3,4}$, S. M. Mari ${ }^{8,19}$, G. Marsella ${ }^{3,4}$, D. Martello ${ }^{3,4}$, S. Mastroianni $^{2}$, P. Montini ${ }^{8,19}$, C. C. Ning ${ }^{12}$, A. Pagliaro ${ }^{16,20}$, M. Panareo ${ }^{3,4}$, B. Panico ${ }^{10,11}$, L. Perrone $^{3,4}$, P. Pistilli $^{8,19}$, F. Ruggieri $^{8}$, P. Salvini ${ }^{7}$, R. Santonico ${ }^{10,11}$, S. N. Sbano ${ }^{3,4}$, P. R. Shen ${ }^{5}$, X. D. Sheng ${ }^{5}$, F. Shi ${ }^{5}$, A. Surdo ${ }^{4}$, Y. H. Tan ${ }^{5}$, P. VAllania ${ }^{21,22}$,

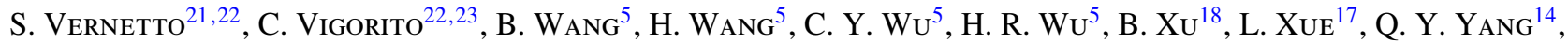

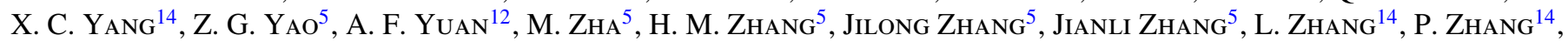

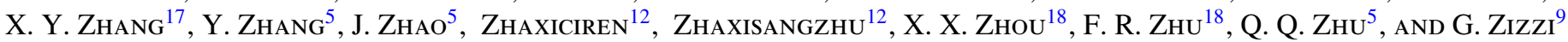

(The ARGO-YBJ COLlaboration)

${ }^{1}$ Dipartimento di Fisica dell'Università di Napoli “Federico II," Complesso Universitario di Monte Sant'Angelo, via Cinthia, I-80126 Napoli, Italy

${ }^{2}$ Istituto Nazionale di Fisica Nucleare, Sezione di Napoli, Complesso Universitario di Monte Sant'Angelo, via Cinthia, I-80126 Napoli, Italy

${ }^{3}$ Dipartimento Matematica e Fisica "Ennio De Giorgi,” Università del Salento, via per Arnesano, I-73100 Lecce, Italy

${ }^{4}$ Istituto Nazionale di Fisica Nucleare, Sezione di Lecce, via per Arnesano, I-73100 Lecce, Italy

${ }^{5}$ Key Laboratory of Particle Astrophysics, Institute of High Energy Physics, Chinese Academy of Sciences, P.O. Box 918, 100049 Beijing, China; chensz@ihep.ac.cn

${ }^{6}$ Dipartimento di Fisica dell’Università di Pavia, via Bassi 6, I-27100 Pavia, Italy

${ }^{7}$ Istituto Nazionale di Fisica Nucleare, Sezione di Pavia, via Bassi 6, I-27100 Pavia, Italy

${ }^{8}$ Istituto Nazionale di Fisica Nucleare, Sezione di Roma Tre, via della Vasca Navale 84, I-00146 Roma, Italy

${ }^{9}$ Istituto Nazionale di Fisica Nucleare-CNAF, Viale Berti-Pichat 6/2, I-40127 Bologna, Italy

${ }^{10}$ Dipartimento di Fisica dell'Università di Roma “Tor Vergata," via della Ricerca Scientifica 1, I-00133 Roma, Italy

${ }^{11}$ Istituto Nazionale di Fisica Nucleare, Sezione di Roma Tor Vergata, via della Ricerca Scientifica 1, I-00133 Roma, Italy

12 Tibet University, 850000 Lhasa, Xizang, China

${ }^{13}$ Hebei Normal University, Shijiazhuang 050016, Hebei, China

${ }^{14}$ Yunnan University, 2 North Cuihu Road, 650091 Kunming, Yunnan, China

15 Dipartimento di Fisica, Università degli Studi di Palermo, Viale delle Scienze, Edificio 18, I-90128 Palermo, Italy

${ }^{16}$ Istituto Nazionale di Fisica Nucleare, Sezione di Catania, Viale A. Doria 6, I-95125 Catania, Italy

${ }^{17}$ Shandong University, 250100 Jinan, Shandong, China

${ }^{18}$ Southwest Jiaotong University, 610031 Chengdu, Sichuan, China

${ }^{19}$ Dipartimento di Fisica dell'Università "Roma Tre," via della Vasca Navale 84, I-00146 Roma, Italy

${ }^{20}$ Istituto di Astrofisica Spaziale e Fisica Cosmica dell'Istituto Nazionale di Astrofisica, via La Malfa 153, I-90146 Palermo, Italy

${ }^{21}$ Osservatorio Astrofisico di Torino dell'Istituto Nazionale di Astrofisica, corso Fiume 4, I-10133 Torino, Italy

${ }^{22}$ Istituto Nazionale di Fisica Nucleare, Sezione di Torino, via P. Giuria 1, I-10125 Torino, Italy

${ }^{23}$ Dipartimento di Fisica dell'Università di Torino, via P. Giuria 1, I-10125 Torino, Italy

Received 2012 November 19; accepted 2013 March 2; published 2013 April 1

\begin{abstract}
We report the observation of a very high energy $\gamma$-ray source whose position is coincident with HESS J1841-055. This source has been observed for 4.5 years by the ARGO-YBJ experiment from 2007 November to 2012 July. Its emission is detected with a statistical significance of 5.3 standard deviations. Parameterizing the source shape with a two-dimensional Gaussian function, we estimate an extension $\sigma=\left(0.40_{-0.22}^{+0.32}\right)^{\circ}$, which is consistent with the HESS measurement. The observed energy spectrum is $d N / d E=(9.0 \pm 1.6) \times 10^{-13}(E / 5 \mathrm{TeV})^{-2.32 \pm 0.23}$ photons $\mathrm{cm}^{-2} \mathrm{~s}^{-1} \mathrm{TeV}^{-1}$, in the energy range $0.9-50 \mathrm{TeV}$. The integral $\gamma$-ray flux above $1 \mathrm{TeV}$ is $1.3 \pm 0.4 \mathrm{Crab}$, which is $3.2 \pm 1.0$ times the flux derived by HESS. The differences in the flux determination between HESS and ARGO-YBJ and possible counterparts at other wavelengths are discussed.
\end{abstract}

Key word: gamma rays: general

Online-only material: color figures

\section{INTRODUCTION}

Very high energy (VHE) $\gamma$-ray astronomy has opened a new window to exploring the extreme non-thermal phenomena in the universe. VHE $\gamma$-rays are tracers of non-thermal particle acceleration and are used to probe the conditions and the underlying astrophysical processes inside their sources. In the past decade, great progress has been made in the field of VHE $\gamma$-ray astronomy. More than 100 VHE $\gamma$-ray emitters have been detected within several categories: active galactic nuclei (AGNs), pulsar wind nebulae (PWNs), supernova remnants (SNRs), X-ray binaries (XBs), and starburst galaxies. However, there is a fraction of VHE sources that remain unidentified because they do not appear to have obvious counterparts at other wavelengths. These kinds of sources may constitute a new class of objects with different emission properties. 
HESS J1841-055 is an enigmatic unidentified VHE $\gamma$-ray source discovered by the HESS collaboration during the Galactic plane survey (Aharonian et al. 2008). Its image shows a high extension, the measured axes for an elongated two-dimensional Gaussian shape being $0.41 \pm 0.04$ (major) and $0.25 \pm 0.02$ (minor). HESS J1841-055, therefore, is one of the most extended sources in the VHE $\gamma$-ray band. The spectrum is best fitted by a simple power law with photon index $\alpha=-2.41 \pm 0.08$ in the energy range $0.54 \mathrm{TeV}$ to $80 \mathrm{TeV}$. The integral flux is $9.1 \times 10^{-12}$ photons $\mathrm{cm}^{-2} \mathrm{~s}^{-1}$ at energies above $1 \mathrm{TeV}$, about $40.3 \%$ of the Crab unit (Aharonian et al. 2006a).

To date, no obvious counterpart has been found at other wavelengths. The wide VHE $\gamma$-ray morphology suggests that HESS J1841-055 may be the blend of multiple sources. Aharonian et al. (2008) found four candidates which could be responsible for at least part of the entire VHE $\gamma$-ray emission: the two pulsars PSR J1841-0524 and PSR J1838-0549, the diffuse source G26.6-0.1, which is a candidate SNR based on its ASCA spectrum, and finally, the high-mass $\mathrm{XB} \mathrm{AX}$ J1841.0-0536. Based on a striking spatial correlation, Sguera et al. (2009) propose that the supergiant fast X-ray transient (SFXT) AX J1841.0-0536 could be responsible for at least a fraction of the VHE $\gamma$-ray emission from HESS J1841-055, thus being the prototype of a new class of Galactic transient $\mathrm{MeV} / \mathrm{TeV}$ emitters. Using $\gamma$-rays with energies $>100 \mathrm{GeV}$ detected by Fermi-LAT, Neronov \& Semikoz (2010) found an event cluster adjacent to HESS J1841-055 and a more extended event cluster at the opposite side. This may be evidence that HESS J1841-055 is composed of at least two different components. On the other hand, Neronov \& Semikoz (2012) suggest an association only with PSR J1841-0524, which is situated in the center of the extended source.

The ARGO-YBJ experiment is an air shower array with a large field of view (FOV) that continuously monitors the northern sky. The emission from the Crab Nebula has been detected with a statistical significance of 17 standard deviations (s.d.) at energies around $1 \mathrm{TeV}$. With such a sensitivity, another four known VHE $\gamma$-ray sources have been detected with significance greater than 5 $\sigma$ : Mrk 421 (Bartoli et al. 2011a), Mrk 501 (Bartoli et al. 2012a), and the two extended sources MGRO J2031+41 (Bartoli et al. 2012b) and MGRO J1908+06 (Bartoli et al. 2012c). It should be pointed out that the fluxes of the two extended sources measured by the extensive air shower (EAS) arrays Milagro and ARGO-YBJ are much higher than that determined by the Cherenkov arrays, showing that there are some systematic differences between the two observation techniques for extended sources (Bartoli et al. 2012b, 2012c; Abdo et al. 2012). Since HESS J1841-055 is also an extended source, its study would benefit from an observation using EAS arrays. HESS J1841-055 is observed by ARGO-YBJ, at the edge of its FOV, $4.8 \mathrm{hr} \mathrm{day}^{-1}$ with a zenith angle less than $50^{\circ}$, culminating at 35.7 . This work presents the observation results for HESS J1841-055 with the ARGO-YBJ experiment.

\section{THE ARGO-YBJ EXPERIMENT}

The ARGO-YBJ experiment is a full coverage EAS array resulting from a collaboration between Chinese and Italian institutions and is designed for VHE $\gamma$-ray astronomy and cosmic-ray observations. The detector is operating at the Yangbajing International Cosmic Ray Observatory (Tibet, China), at an altitude of $4300 \mathrm{~m}$ a.s.l. The detector, extensively described in Aielli et al. (2006, 2009c), consists of a single layer of resistive plate chambers (RPCs; $2.8 \mathrm{~m} \times 1.25 \mathrm{~m}$ ), equipped with 10 logical pixels (called "pads," $55.6 \mathrm{~cm} \times 61.8 \mathrm{~cm}$ ) used for triggering and timing purposes. One hundred and thirty clusters (each composed of 12 RPCs) are installed to form the central carpet of $74 \mathrm{~m} \times 78 \mathrm{~m}$ with an active area of $\sim 93 \%$, surrounded by 23 additional clusters ("guard ring"). The total area of the array is $110 \mathrm{~m} \times 100 \mathrm{~m}$. The arrival time of the particles is measured by time-to-digital converters (TDCs) with a resolution of about 1.8 ns (Aielli et al. 2009c). To calibrate the 18,360 TDC channels, a software method has been developed using cosmic-ray showers (He et al. 2007). The calibration precision is $0.4 \mathrm{~ns}$ and the procedure is applied every month (Aielli et al. 2009a).

The central 130 clusters started taking data in 2006 July, while the complete ARGO-YBJ detector including the "guard ring" has collected data since 2007 November. The RPC carpet is connected to two independent data acquisition systems, corresponding to the shower and scaler operation modes (Aielli et al. 2008). In this work, only data from the shower mode are used. In the shower mode, the ARGO-YBJ detector is operated by requiring at least 20 fired pads $\left(N_{\text {pad }}\right)$ within $420 \mathrm{~ns}$ on the entire carpet detector. The trigger rate is $3.5 \mathrm{kHz}$ with a dead time of $4 \%$ and the average duty cycle is higher than $86 \%$.

The high granularity of the apparatus allows a complete and detailed space-time three-dimensional reconstruction of the shower profile and therefore of the incident direction of the primary particle. Through the analysis of the position, size, and shape of the reconstructed Moon and Sun shadows in the cosmic-ray flux, the angular resolution, pointing accuracy, and stability of the ARGO-YBJ detector array have been thoroughly tested (Bartoli et al. 2011b; Aielli et al. 2011). The point-spread function (PSF) is quantified using a parameter $\psi_{70}$ as the opening angle containing $71.5 \%$ of the events. For cosmic-ray-induced air showers $\psi_{70}$ is 2.8 for $N_{\text {pad }} \sim 20$, while it becomes 0.47 for $N_{\text {pad }}>1000$ (Bartoli et al. 2011a, 2011b), in good agreement with Monte Carlo predictions. The simulations show that the angular resolution for $\gamma$-induced showers is $30 \%-40 \%$ smaller. The effective area of the detector for $\gamma$-induced showers depends on the $\gamma$-ray energy and incident zenith angle, e.g., it is about $100 \mathrm{~m}^{2}$ at $100 \mathrm{GeV}$ and $>10,000 \mathrm{~m}^{2}$ above $1 \mathrm{TeV}$ for a zenith angle of $20^{\circ}$ (Aielli et al. 2009b).

\section{DATA ANALYSIS}

The data set used in this analysis refers to the period from 2007 November to 2012 July. The total effective observation time is 1492.6 days. For the analysis presented in this paper, only events with a zenith angle less than $50^{\circ}$ are used, and the data set is divided into six groups according to $N_{\text {pad }}$. To achieve a good angular resolution, the event selections used in Bartoli et al. (2011a) are applied here. After filtering the total number of events used in this work is $2.42 \times 10^{11}$. The opening angles $\psi_{70}$ for events with $N_{\text {pad }}>60$ and $N_{\text {pad }}>100$ are 1.36 and 0.98 , respectively. For the data set in each group, the whole sky map in celestial coordinates (right ascension and declination) is divided into a grid of $0.1 \times 0.1$ bins and filled with detected events according to their reconstructed arrival direction. The "direct integral method" (Fleysher et al. 2004) is adopted to estimate the cosmic-ray background and to extract the excess of $\gamma$-induced showers from each bin. The correction procedure described in Bartoli et al. (2011a) has been applied to remove the effect of cosmic-ray anisotropy on a scale of $11^{\circ} \times 11^{\circ}$. A Gaussian smoothing method is used to take into account the PSF of the ARGO-YBJ detector. That is, the events in a circular area centered on the bin with an angular radius of $1.3 \psi_{70}$ are summed after weighting with the Gaussian-shaped 


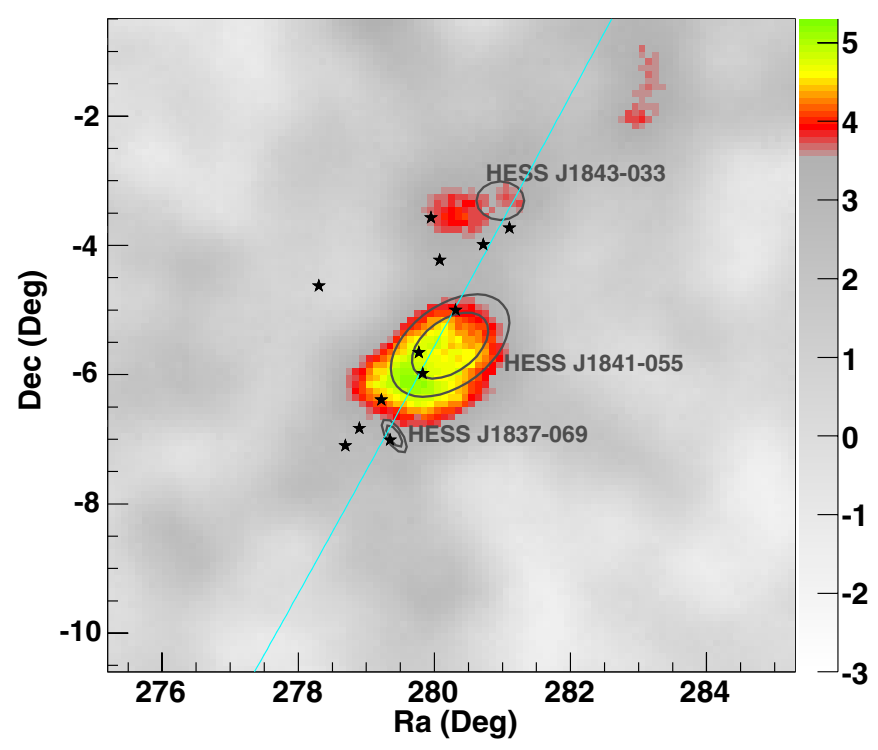

Figure 1. Significance map around HESS J1841-055 as observed by the ARGO-YBJ experiment. The two ellipses for HESS J1841-055 and HESS J1837-069 indicate their positions and the $68 \%$ and $90 \%$ contours of their extension regions (Aharonian et al. 2008). The position and possible extension of HESS J1843-33 are marked with ellipses (Hoppe 2007). The stars mark the location of the GeV $\gamma$-ray sources around HESS J1841-055 in the second Fermi-LAT catalog (Nolan et al. 2012). The solid line indicates the Galactic plane.

(A color version of this figure is available in the online journal.)

PSF. The Li-Ma method ( $\mathrm{Li} \&$ Ma 1983) is used to estimate the significance of the excess in each bin.

With this procedure, the northern sky has been surveyed (Cao $\&$ Chen 2011). The significance of the excess observed from the direction of the Crab Nebula is 17 s.d., indicating that the cumulative 5 s.d. sensitivity of ARGO-YBJ has reached $0.3 \mathrm{Crab}$ for point sources. The sensitivity is dependent on the declination of the source, being degraded by a factor of 3.5 at the declination of HESS J1841-055 (Cao \& Chen 2011). For an extended source with a symmetrical two-dimensional Gaussian shape with $\sigma=0.40$, the sensitivity is degraded by $15 \%$. Therefore, a simple estimation indicates that the flux from HESS J1841-055 should be about 1.2 Crab in order to be detected by ARGO-YBJ with 5 s.d. The required flux slightly varies if the spectrum is different from that of the Crab Nebula.

\section{RESULTS}

The significance map around HESS J1841-055, as observed by ARGO-YBJ using events with $N_{\text {pad }}>60$, is shown in Figure 1. For comparison, the 12 sources in the second FermiLAT catalog (Nolan et al. 2012) around HESS J1841-055 are also marked in the figure. Weak excesses are observed along the Galactic plane, indicating a diffuse $\gamma$-ray emission. An analysis of the diffuse $\gamma$-ray emission using ARGO-YBJ data can be found in Ma (2011). The highest significance is 5.3 s.d. at $\alpha=18^{\mathrm{h}} 39^{\mathrm{m}}$ and $\delta=-6^{\circ} 3^{\prime}(\mathrm{J} 2000)$, which is displaced 0.7 from the center of HESS J1841-055. To estimate the statistical error of the position, the data are sampled 20,000 times and the statistical errors in both directions are about 0.45 . However, most of the excesses overlap the extended region of HESS $\mathrm{J} 1841-055$ and its gravity center $\left(\alpha=18^{\mathrm{h}} 40^{\mathrm{m}} \pm 12^{\mathrm{m}}\right.$ and $\delta=$ $\left.-5^{\circ} 52^{\prime} \pm 13^{\prime}\right)$, obtained using all the pixels with significance greater than 3 s.d. within $3^{\circ} \times 3^{\circ}$ around HESSJ1841-055, is 0.4 off-center of HESS J1841-055. These displacements may be caused by different concurring effects besides fluctuation. (1) One possible cause is complex morphology. According to the HESS result, HESS J1841-055 possibly has two or three peaks and the positions of the two largest ones are both 0.44 off-center. (2) Another possible cause is the systematic pointing error of ARGO-YBJ which is 0.2 , slightly increasing at the boundary of the ARGO-YBJ FOV. (3) A third possible cause is the contribution of the nearby VHE source HESS J1837-069, partially containing its emission. Therefore, the signal position observed by ARGO-YBJ largely overlaps HESS J1841-055.

The intrinsic extension of HESS J1841-055 is determined by fitting the distribution of $\theta^{2}$ for the events exceeding the background as shown in Figure 2, where $\theta$ is the angular distance of each event to the position of HESS J1841-055. To achieve a good angular resolution, only events with $N_{\text {pad }}>100$ are used in this fit. In order to fit the data, a set of $\gamma$-rays is generated taking into account the spectral energy distribution (SED), the intrinsic source extension, and the detector PSF. The extension is estimated by minimizing the $\chi^{2}$ between data and generated events, from $0^{\circ}$ to $1^{\circ}$ with steps of 0.1 . Assuming a spectral index of -2.3 , the intrinsic extension is determined to be $\sigma_{\text {ext }}=\left(0.40_{-0.22}^{+0.32}\right)^{\circ}$. It is found that the dependence on the SED is negligible within the uncertainties. This result is consistent with the estimation by the HESS collaboration, i.e., $0.41 \pm 0.04$ and $0.25 \pm 0.02$ along the major and minor axes, respectively (Aharonian et al. 2008).

Assuming an intrinsic extension $\sigma_{\text {ext }}=0.40$, we estimate the spectrum of HESS J1841-055 using the ARGO-YBJ data with the conventional fitting method described in Bartoli et al. (2011a). In this procedure, the expectation function is generated by sampling events in the energy range $10 \mathrm{GeV}$ to $100 \mathrm{TeV}$ and taking into account the detailed ARGO-YBJ detector response, assuming a power law with its spectral index as a parameter. We define five $N_{\text {pad }}$ intervals: 60-59, 100-199, 200-499, 500-999, and $\geqslant 1000$. The best fit to the SED and the corresponding $1 \sigma$ error region are shown in Figure 3. The differential flux $\left(\mathrm{TeV}^{-1} \mathrm{~cm}^{-2} \mathrm{~s}^{-1}\right)$ in the energy range from $0.9 \mathrm{TeV}$ to $50 \mathrm{TeV}$ is

$$
\frac{d N}{d E}=(9.0 \pm 1.6) \times 10^{-13}(E / 5 \mathrm{TeV})^{-2.32 \pm 0.23}
$$

The median energies of the five $N_{\text {pad }}$ intervals are 2.3, 3.5, 7.1, 14, and $22 \mathrm{TeV}$, respectively. The integral flux is $1.3 \pm 0.4$ Crab at energies above $1 \mathrm{TeV}$, which is $3.2 \pm 1.0$ times the flux determined by the HESS experiment, i.e., 0.40 Crab.

\section{DISCUSSION}

The integrated energy flux above $1 \mathrm{TeV}$ measured by ARGOYBJ is $\sim 1.9 \times 10^{-10} \mathrm{erg} \mathrm{cm}^{-2} \mathrm{~s}^{-1}$, corresponding to a source luminosity, assuming isotropic emission, of $L(>1 \mathrm{TeV}) \sim 2.3 \times$ $10^{34}\left(D / 1 \mathrm{kpc}^{2} \mathrm{erg} \mathrm{s}^{-1}\right.$, where $D$ is the distance to the source. However, due to the limitations in the angular resolution, this flux may also include other contributions apart from HESS J1841-055. Diffuse $\gamma$-rays, produced by cosmic rays interacting with matter in the Galaxy plane, are expected to contribute to the ARGO-YBJ result. According to the measurement of diffuse $\gamma$-ray flux from the inner Galactic plane using ARGO-YBJ data (Ma 2011), this contribution to the flux from HESS J1841-055 in the five intervals is less than $4 \%$. Assuming the HESS shape for the source instead of the symmetrical two-dimensional Gaussian shape, the flux would only vary $2.2 \%$. HESS J1837-069 is the nearest VHE $\gamma$-ray source with an angular distance of 


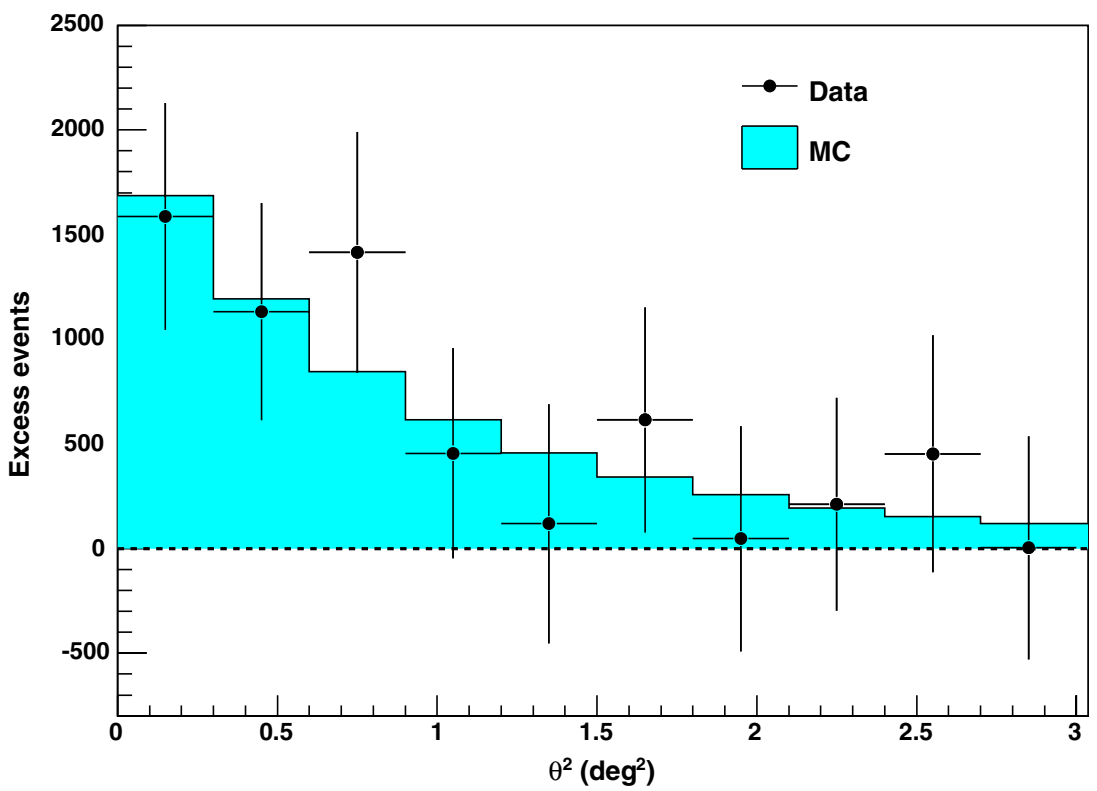

Figure 2. Distribution of $\theta^{2}$ for the number of excess events around HESS J1841-055. The filled region outlines the best fit to simulated data assuming a symmetrical two-dimensional Gaussian shape with $\sigma=0.40$.

(A color version of this figure is available in the online journal.)

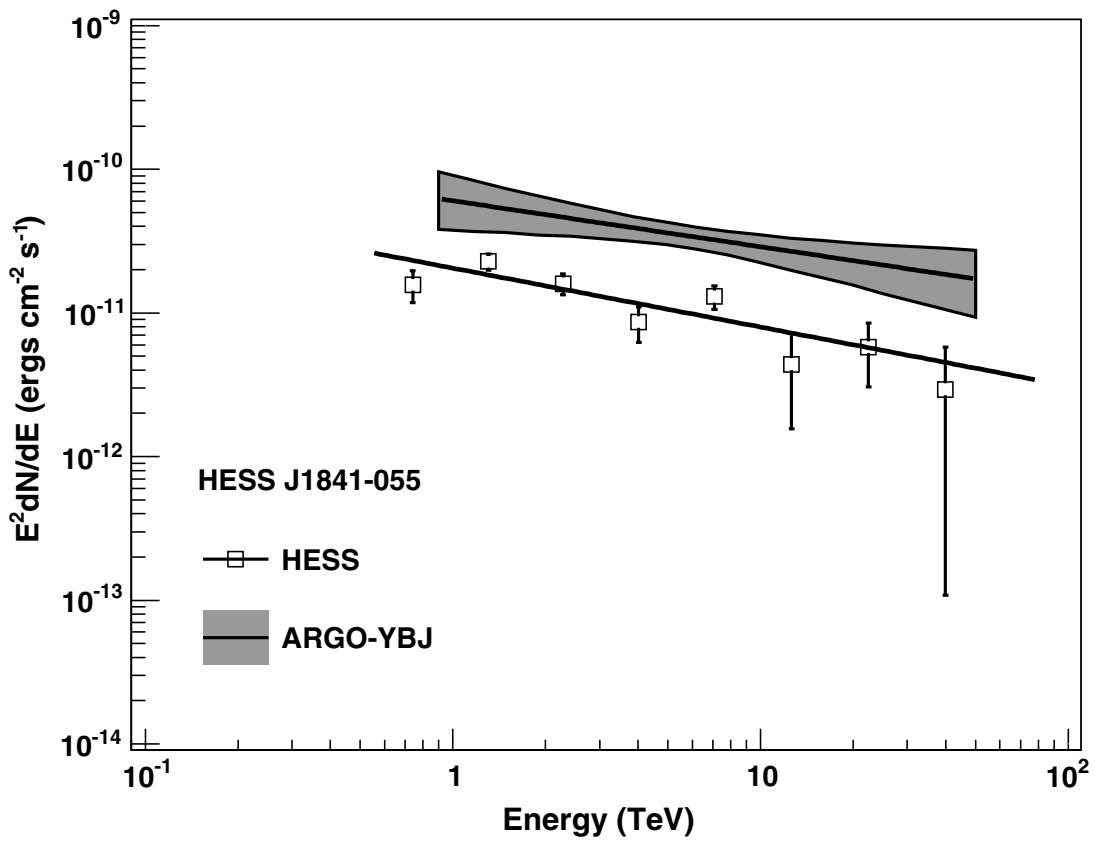

Figure 3. Energy density spectrum of HESS J1841-055 as measured by the ARGO-YBJ experiment: the solid line and shaded area indicate the differential energy spectrum and the 1 s.d. error region. The spectrum measured by HESS (Aharonian et al. 2008) is also reported for comparison. Only statistical errors are shown.

1.62. The flux from HESS J1837-069 at energies above $1 \mathrm{TeV}$ is $17 \%$ that of the Crab with spectral index -2.27 (Aharonian et al. 2006b). Its contributions to the five intervals are estimated to be $5.8 \%, 2.7 \%, 1.0 \%, 0.7 \%$, and $0.2 \%$, respectively. The second nearest source is HESS J1843-033, whose flux is still unknown. A hot spot with a marginal significance of 4.1 s.d. is observed near its position (Hoppe 2007). With an angular separation of 2.6 , its contribution is estimated to be lower compared with HESS J1837-069. The contribution from other known VHE $\gamma$-ray sources is negligible. Moreover, an estimate of the systematic error of ARGO-YBJ is described in Bartoli et al. (2012b). With an incomplete list of possible causes, such as time resolution variation, event rate variation with environment parameters, and pointing error, the systematic error for point sources is found to be less than $30 \%$, and is lower for extended sources. Thus, the systematic error of ARGO-YBJ alone is not enough to explain the discrepancy.

HESS J1841-055 is observed by ARGO-YBJ only at high zenith angles $\left(\theta>35^{\circ} 7\right)$, while the observation of the Crab is also possible at low zenith angles. This difference may cause some systematic errors when estimating the spectrum of HESS J1841-055. To check a possible systematic error, observations of the $\mathrm{Crab}$ at zenith angles higher than $30^{\circ}$ are used. With this selection, the average zenith angle is about the same as that of HESS J1841-055. The result is that the Crab spectral index varies from $(2.58 \pm 0.07)$ to $(2.52 \pm 0.21)$, and the flux above 


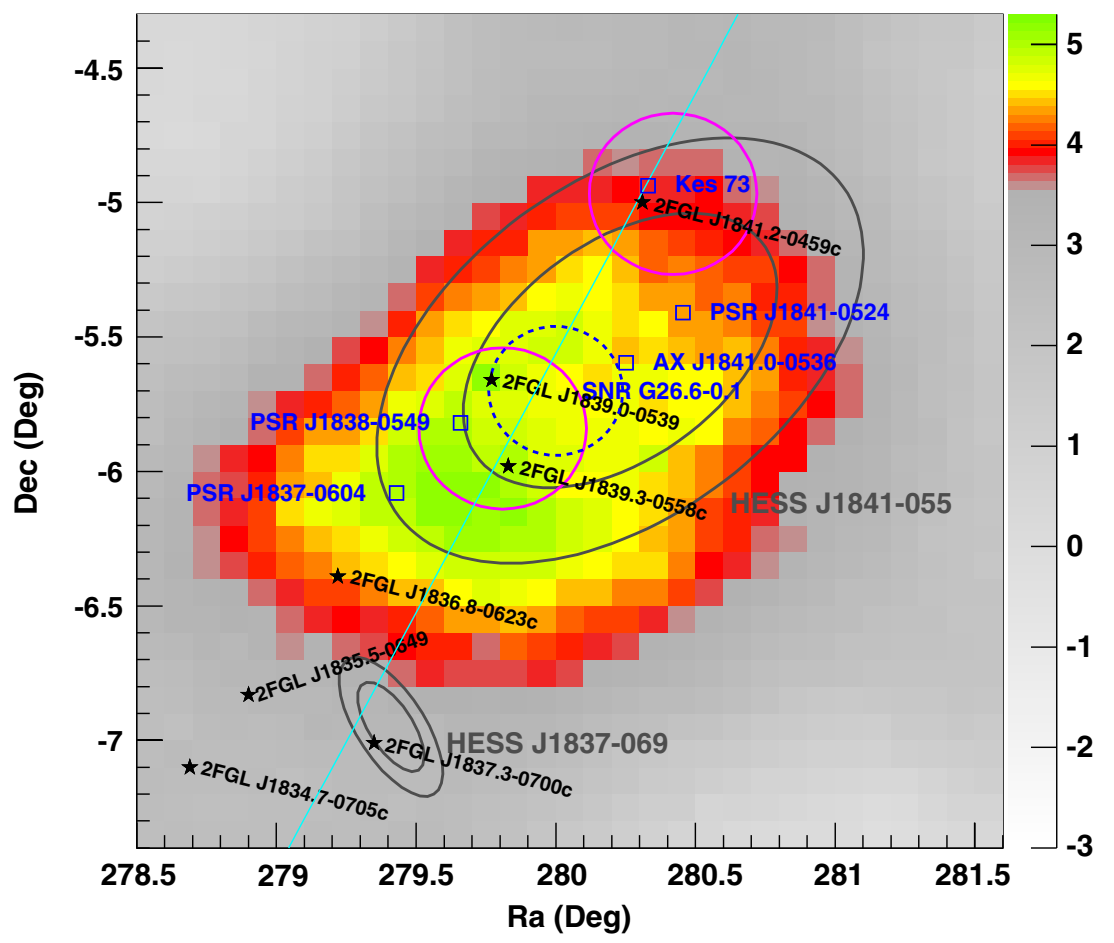

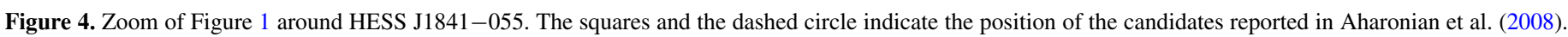

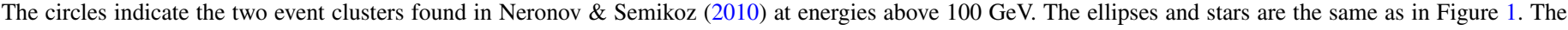
solid line indicates the Galactic plane.

(A color version of this figure is available in the online journal.)

$1 \mathrm{TeV}$ is $(35 \pm 28) \%$ higher. Due to the large statistical error, we cannot exclude a systematic effect causing the difference of flux. However, even taking this systematic error into account, the flux of HESS J1841-055 observed by ARGO-YBJ is still about twice that determined by HESS.

On the other hand, the discrepancy is similar to that found for the two extended sources MGRO J1908+06 and MGRO J2031+41 (Bartoli et al. 2012c, 2012b). The fluxes measured by the EAS arrays Milagro and ARGO-YBJ are much higher than that determined by the Cherenkov arrays. Since a good agreement has been achieved on the "standard candle", the Crab Nebula, some systematic differences between the two techniques should exist only for extended sources. As pointed out by Abdo et al. (2012), due to their limited FOV, Cherenkov telescopes might count the extended emission as background, especially when using the "wobble mode" to estimate the latter. It is worth noting that the "wobble mode" was used when HESS observed HESS J1841-055, and the source was offset by 0.7 (Aharonian et al. 2008). The "reflected-region technique" is used to estimate the background for spectra in a region partially overlapping the extended source. As a result, HESS would measure an emission fainter than that measured by ARGO-YBJ.

Different scenarios have been proposed to explain the emission mechanism of $\mathrm{TeV}$ photons. VHE $\gamma$-rays can be produced via inverse-Compton of background photon fields by high-energy electrons, or, in hadronic models, by inelastic proton-proton or proton-photon interactions. In both scenarios, X-ray and radio synchrotron emissions are expected, therefore the lack of a low-energy counterpart for HESS J1841-055 poses a question about the nature of the emission mechanism. Aharonian et al. (2008) searched for counterparts responsible for the VHE $\gamma$-ray emission and discussed the possible association with six candidates, marked in Figure 4, which is a zoom of
Figure 1 around HESS J1841 -055 . Three of them are the pulsars PSR J1838-0549, PSR J1841-0524, and PSR J1837-0604, of which only the last has a high enough spin-down flux $\left(\dot{E} / D^{2}=5.2 \times 10^{34} \mathrm{erg} \mathrm{s}^{-1} \mathrm{kpc}^{-2}\right)$ to be a counterpart candidate. This source is at the boundary of the HESS region, but not far from the center of gravity of the ARGO-YBJ signal. However, as pointed out in Aharonian et al. (2008), since the $\mathrm{TeV}$ emission is usually attributed to a relic population of electrons, some contribution can also be expected from the other pulsars if they had a much higher spin-down luminosity in the past. No cataloged PWNs at longer wavelengths are associated with these pulsars, however, according to the recent calculations of Tibolla et al. (2012); during their evolution ancient PWNs ( $\gg 10 \mathrm{kyr}$ ) might appear as $\mathrm{GeV}-\mathrm{TeV} \gamma$-ray sources without X-ray counterparts. The three other cataloged objects located inside the HESS uncertainty region are the SNR G027.4 (also known as Kes73), the high-mass XB AX J1841.0-0536, and the diffuse source G26.6-0.1. The SNR Kes73 lies at the edge of the $\mathrm{TeV}$ emission region. The point-like nature of $\mathrm{AX}$ J1841.0-0536, the only soft $\gamma$-ray source detected within the HESS J1841-055 error ellipse, its variability, and the required luminosity (about $10^{36} \mathrm{erg} \mathrm{s}^{-1}$ according to the ARGO-YBJ data and assuming a distance of $6.9 \mathrm{kpc}$ as inferred in Sguera et al. 2009) exclude its association with the entire emission from the extended HESS source. Also the diffuse source G26.601 , at only $1.3 \mathrm{kpc}$ and well inside the emission region, could be responsible at least for part of the $\mathrm{TeV}$ flux. In Figure 4 four $\mathrm{GeV} \gamma$-ray sources from the Fermi-LAT second source catalog within the extension of HESS J1841-055 are also shown: 2FGL J1839.3-0558c, 2FGL J1836.8-0623c, and the two diffuse sources 2FGL J1839.0-0539 and 2FGL J1841.2-0459c (Nolan et al. 2012). Moreover, the two event clusters at energies above $100 \mathrm{GeV}$ found by Neronov \& Semikoz (2010) are shown. 
Three GeV sources are within the two event clusters, suggesting that they may also be VHE emitters: 2FGL J1841.2-0459c is coincident with the SNR Kes 73 while 2FGL J1839.3-0558c and 2FGL J1839.0-0539 are spatially associated with PSR J1838-0549 and the diffuse X-ray source G26.6-0.1, respectively. As remarked in Tibolla et al. (2012), the recent observation by Fermi-LAT of GeV sources not firmly associated with $\mathrm{X}$-ray counterparts suggests that unidentified VHE sources can be explained as ancient PWNs.

A hadronic scenario is proposed in Neronov \& Semikoz (2012). These authors consider the extended $\gamma$-ray emission produced by high-energy cosmic rays escaping from the source and diffusing in the interstellar medium (ISM). The $\gamma$-ray emission should result from the interaction of these cosmic rays with ISM particles. Such extended emission regions should be visible as VHE $\gamma$-ray sources with fluxes of order $10^{-11} \mathrm{erg} \mathrm{cm}^{-2} \mathrm{~s}^{-1}$ above $100 \mathrm{GeV}$. From the analysis of the Fermi-LAT data they suggest the young nearby pulsar PSR J1841-0524 as a possible low-energy counterpart to HESS J1841-055. However, as already stated, due to the energy balance, this association is not without problems. The proton/nuclei contribution to the extended $\gamma$-ray flux should generate a comparable flux of $\mathrm{TeV}$ neutrinos with a spectrum expected to follow the $\gamma$-ray spectrum. Thus, the observation of high-energy neutrinos from the HESS source could provide a crucial test of this model. A search for individual neutrino sources over a large fraction of both the northern and southern skies has been carried out by the IceCube detector in the 40 string configuration (Abbasi et al. 2011). The large background from atmospheric muons reduces the IceCube sensitivity to neutrino sources in the southern sky at $\mathrm{TeV}$ energies, thus the derived upper limits are not stringent enough to constrain the hadronic scenario. Data from the combined operation of IceCube and AMANDA have been used to scan for sources in the Galactic plane (Abbasi et al. 2013) with a neutrino flux sensitivity of about $10^{-11}-10^{-12} \mathrm{erg} \mathrm{cm}^{-2} \mathrm{~s}^{-1}$ at $\mathrm{TeV}$ energies. However, the surveyed range of Galacic longitude $\left(36^{\circ}<l<210^{\circ}\right)$ does not include the region where HESS $\mathrm{J} 1841-055$ is located.

In the case of hadronic scenarios one expects the source extension to be much larger than seen by Cherenkov telescopes (up to the degree scale). Therefore, the lower angular resolution and the large FOV of ARGO-YBJ allow the collection of photons from a larger source area. This could partially explain the discrepancy in flux with the HESS Cherenkov telescope.

Recently, Giacinti et al. (2012) found that the diffusion of cosmic rays and electrons around point sources is strongly anisotropic and shows filamentary structures, which may cause a shift of the centroid position between HESS and ARGO-YBJ.

\section{CONCLUSIONS}

Since 2007 November the ARGO-YBJ experiment has been monitoring the northern sky at $\mathrm{TeV}$ photon energies via the high duty cycle. Using data up to 2012 July, an excess with statistical significance of 5.3 s.d. is detected from the direction of the unidentified source HESS J1841-055. The source location and extension are consistent with those determined by HESS: however, the measured flux above $1 \mathrm{TeV}$ is about three times higher. This discrepancy, already found in the observation of other extended sources, could originate from the different techniques used in the background estimation for extended sources with ARGO-YBJ and HESS data. The extended morphology of HESS J1841-055 and the presence of several sources within the $90 \%$ confidence error region suggest contributions from more than one of them, but so far no clear counterparts in lower-energy wavebands can be identified. However, the possibility of a $\mathrm{GeV}-\mathrm{TeV} \gamma$-ray source without any counterpart cannot be excluded. Both leptonic and hadronic productions of $\gamma$-rays have been proposed, but it is not easy to distinguish between the two contributions based only on the $\gamma$-ray data. The current upper limits to the neutrino flux from the HESS J1841-055 region are too high to test the hadronic model. Further multiwavelength observations from radio to $\mathrm{GeV}$ energies and data from neutrino telescopes of suitable sensitivity are needed in order to disentangle the different emission possibilities.

This work is supported in China by the NSFC (Nos. 10120130794 and 11205165), the Chinese Ministry of Science and Technology, the Chinese Academy of Sciences, the Key Laboratory of Particle Astrophysics, CAS, and in Italy by the Istituto Nazionale di Fisica Nucleare (INFN).

We also acknowledge the essential support of W. Y. Chen, G. Yang, X. F. Yuan, C. Y. Zhao, R. Assiro, B. Biondo, S. Bricola, F. Budano, A. Corvaglia, B. D’Aquino, R. Esposito, A. Innocente, A. Mangano, E. Pastori, C. Pinto, E. Reali, F. Taurino, and A. Zerbini in the installation, debugging, and maintenance of the detector.

\section{REFERENCES}

Abbasi, R., Abdou, Y., Abu-Zayyad, T., et al. 2011, ApJ, 732, 18

Abbasi, R., Abdou, Y., Ackermann, M., et al. 2013, ApJ, 763, 33

Abdo, A. A., Abeysekara, U., Allen, B. T., et al. 2012, ApJ, 753, 159

Aharonian, F., Akhperjanian, A. G., Barres de Almeida, U., et al. 2008, A\&A, 477,353

Aharonian, F., Akhperjanian, A. G., Bazer-Bachi, A. R., et al. 2006a, A\&A, 457, 899

Aharonian, F., Akhperjanian, A. G., Bazer-Bachi, A. R., et al. 2006b, ApJ, 636, 777

Aielli, G., Assiro, R., Bacci, C., et al. 2006, NIMPA, 562, 92

Aielli, G., Bacci, C., Barone, F., et al. 2008, APh, 30, 85

Aielli, G., Bacci, C., Bartoli, B., et al. 2009a, APh, 30, 287

Aielli, G., Bacci, C., Bartoli, B., et al. 2009b, APh, 32, 47

Aielli, G., Bacci, C., Bartoli, B., et al. 2009c, NIMPA, 608, 246

Aielli, G., Bacci, C., Bartoli, B., et al. 2011, ApJ, 729, 113

Bartoli, B., Bernardini, P., Bi, X. J., et al. 2011a, ApJ, 734, 110

Bartoli, B., Bernardini, P., Bi, X. J., et al. 2011b, PhRvD, 84, 022003

Bartoli, B., Bernardini, P., Bi, X. J., et al. 2012a, ApJ, 758, 2

Bartoli, B., Bernardini, P., Bi, X. J., et al. 2012b, ApJL, 745, L22

Bartoli, B., Bernardini, P., Bi, X. J., et al. 2012c, ApJ, 760, 110

Cao, Z., \& Chen, S. Z. 2011, in Proc. 32nd ICRC, http://icrc2011.ihep.ac.cn Fleysher, R., Fleysher, L., Nemethy, P., \& Mincer, A. I. 2004, ApJ, 603, 355

Giacinti, G., Kachelrieß, M., \& Semikoz, D. V. 2012, PhRvL, 108, 261101

He, H. H., Bernardini, P., Calabrese Melcarne, A. K., \& Chen, S. Z. 2007, APh, 27,528

Hoppe, S. 2007, in Proc. 30th ICRC, ed. R. Caballero et al. (Mexico City: Univ. Nacional Autonoma de Mexico), 579

Li, T. P., \& Ma, Y. Q. 1983, ApJ, 272, 317

Ma, L. L. 2011, in Proc. 32nd ICRC, http://icrc2011.ihep.ac.cn

Neronov, A., \& Semikoz, D. 2010, arXiv:1011.0210

Neronov, A., \& Semikoz, D. 2012, PhRvD, 85, 083008

Nolan, P. L., Abdo, A. A., Ackermann, M., et al. 2012, ApJS, 199, 31

Sguera, V., Romero, G. E., Bazzano, A., et al. 2009, ApJ, 697, 1194

Tibolla, O., Vorster, M., de Jager, O., et al. 2012, in AIP Conf. Proc. 1505, High Energy Gamma Ray Astronomy, ed. F. A. Aharonian, W. Hofmann, \& F. M. Rieger (Melville, NY: AIP), 349 